\title{
Les frontières sociolinguistiques du sujet : disons, c'est-à-dire, je veux dire
}

\author{
Claudine Moïse \\ Université Grenoble Alpes
}

La question des frontières est au cœur de la réflexion sociolinguistique. La figure de la frontière n'est pas seulement une ligne de séparation, une démarcation, une limite ; elle induit le franchissement et la mobilité. Elle renvoie à des jeux ambivalents entre liaisons et séparations, transitions et ruptures, dehors et dedans, protection et disqualification, enfermement et transgression, inclusion et exclusion. Dans la perspective d'une sociolinguistique des contacts de langues ${ }^{1}$, la frontière est considérée dans son extériorité au sujet parlant ${ }^{2}$, construction entre les Etats et les territoires, entre les groupes, les communautés de pratiques (Lave et Wenger 1991) et de parole (Hymes 1972). En tant que frontière ethnolinguistique (Barth 1969), elle est constitutive de cette sociolinguistique et des façons d'appréhender les pratiques et la variabilité langagières, quand elle renseigne sur les processus sociaux d'inclusion et d'exclusion, de domination, de normes et d'inégalité en jeu dans le champ social. La frontière peut alors s'appréhender en fonction des majorités et minorités dans des rapports de pouvoir, ou à travers des identités sociales qui s'élaborent et se mettent en scène par les pratiques langagières.

En allant dans une autre direction, peut-être moins attendue et entendue, celle d'une sociolinguistique du sujet, j'ai choisi ici de me pencher sur les frontières symboliques intérieures de l'individu pris dans la complexité de ses identités en changement et en mouvement dans un contexte social. Les déplacements intérieurs à soi peuvent alors se dire par des marques langagières subjectives, d'hybridation, d'informatisation, de stylisation, de jeux de normes, mais aussi, comme ce sera le cas ici, par des indices de reformulation, tels que les formes disons, c'est-à-dire, je veux dire.

Je voudrais explorer ces formes de ruptures et de franchissements, qui se dessinent donc à travers le sujet psychique en prise avec sa propre énonciation, le sujet en interaction avec un

\footnotetext{
${ }^{1}$ En France, historiquement, la sociolinguistique du contact des langues et du plurilinguisme s'est développée dans les années 1970 avec les créolistes et dans la mouvance des études sur la diglossie occitane. Elle est l'héritière des approches sur les langues minoritaires dans des perspectives aménagementistes et diglossiques sans s'inscrire dans une conception à tendance structuraliste de la langue comme l'est la sociologie du langage prise dans les traces de Fishman en Amérique du Nord. Elle s'est définie aussi depuis Weinreich autour des recherches sur le bilinguisme individuel et sociétal et, plus tard, autour de l'alternance de codes et de l'ethnographie de la communication. Aujourd'hui, en prise avec les phénomènes de mondialisation elle s'est largement étendue dans son acception (Simonin et Wharton 2013), du plurilinguisme à la sociolinguistique de la migration par exemple.

2 À mon sens, cette sociolinguistique du contact se caractérise par le fait qu'elle considère avant tout le sujet dans sa dimension d'appartenance ethnique et sociale sans considérer ni sa part individuelle ni sa part psychique.
} 
autre, un $t u$ qui le révèle à lui-même et pour lequel il se donne à voir. Mais dans le même temps, il s'agira de voir comment ces formes apparaissent quand le sujet cherche à affirmer des positionnements sociaux. Ce balancement intérieur ne serait donc pas seulement quête de soi mais, en même temps, quête d'un rôle social. En ce sens, il s'agit de saisir le sujet intime et le sujet social, l'un n'allant pas sans l'autre, ces va-et-vient intérieurs, de soi au sujet social, rendant compte de «frontières sociolinguistiques ». Cette réflexion autour des frontières m'a donc amenée à interroger, à travers des entretiens, le sujet à la fois comme acteur social et dans son intimité, c'est-à-dire comme participant à des changements sociaux et à des mouvements intérieurs, et de ne pas laisser son traitement aux seules linguistiques du discours ou de l'énonciation.

\section{L'entretien sociolinguistique, lieu de construction du sujet}

J'ai relu pour ce texte un certain nombre d'entretiens que j'ai menés pour différentes recherches ces dernières années. Ces entretiens font partie d'études ethnographiques qui ont pour objet de saisir les changements sociaux et langagiers ${ }^{3}$ auxquels participent les sujets en position minoritaire ou marginale, et ce à travers leur histoire de vie, et donc leurs engagements sociaux et leurs positionnements identitaires. Si dans les recherches en question ${ }^{4}$ il s'agissait de voir comment se manifestaient discursivement les changements sociaux en cours, essentiellement à travers l'analyse des représentations et des discours «sur», j'ai regardé plus précisément ici comment s'exprimait l'investissement personnel, subjectif et social, des locuteurs.

J'ai sélectionné 4 extraits d'entretiens provenant de 3 recherches différentes, extraits qui m'apparaissaient particulièrement pertinents du point de vue de leur mise en discours réflexive. Le premier entretien sur lequel je m'appuie concerne Noël traducteur du nord de 1'Ontario, interviewé en 2003 dans le cadre du projet Prise de Parole $I I^{5}$. Dans la perspective d'une sociolinguistique critique $^{6}$ (Heller 2002) et du contact, « il s'agissait dans ce programme de recherche, d'examiner l'impact de la mondialisation et de la nouvelle économie sur la valeur accordée aux compétences langagières en français, en anglais, et en d'autres langues

\footnotetext{
3 J'entends par «changement social », le processus qui rend compte de transformations de certains phénomènes de société : par exemple le rapport entre majorité et minorité au Canada, la légitimation du plurilinguisme, la valorisation de l'origine ethnique...

${ }^{4}$ Un projet portait sur les changements identitaires des sujets minoritaires au Canada français, en Ontario, face la mondialisation, un autre sur le choix des prénoms dans les couples mixtes franco-arabes, le dernier sur la construction de la violence verbale.

${ }^{5}$ Projet subventionné par le Conseil de recherche en sciences humaines du Canada et par l'Ambassade du Canada. Chercheurs principaux : Monica Heller et Normand Labrie, Université de Toronto (2000-2004).

${ }^{6} \mathrm{La}$ sociolinguistique critique repose sur une démarche ethnographique et en filiation avec l'ethnographie de la communication et la Critical Discourse Analysis.
} 
ainsi que sur les liens entre les compétences langagières, l'identité, les frontières ethnolinguistiques et les rapports de pouvoir et d'inégalité au Canada francophone ${ }^{7}$ ». Les deux autres entretiens explorés concernent Pierre et Fatima, puis Hassan et Françoise, deux couples mixtes français-arabes, interviewés en 2002. À travers le choix des prénoms des enfants dans les couples mixtes franco-arabes, nous avions cherché les parcours et les confrontations identitaires des parents en regard de la situation migratoire en France ${ }^{8}$. Enfin, j’ai analysé un entretien avec un surveillant de collège, Juan, interviewé en 2005 , qui avait participé à notre enquête ethnographique sur la violence verbale dans une ville du sud de la France, enquête d'une année à raison d'une journée par semaine où nous avions suivi une classe de sixième' Ce projet visait à cerner les processus sociaux et interactionnels de la violence verbale en milieu scolaire.

Il est certain que les entretiens sociolinguistiques sont un genre particulier d'interactions. Les chercheurs tentent souvent d'obtenir, par exemple, sous forme de récits de vie, des représentations sur les langues, les pratiques et expériences langagières et identitaires. Ils vont alors faire apparaître le sujet dans ses (re)compositions identitaires à travers mises en récit de soi, narrations et argumentations explicatives. L'intersubjectivité s'exprime, dans la situation d'entretien et dans des interactions sollicitées, par des propos qui n'auraient peut-être jamais été exprimés autrement. Pour ce texte, les extraits d'entretien où apparaissent les particules discursives disons, je veux dire et c'est-à-dire, portent sur des argumentations explicatives, des positionnements identitaires et appréciatifs, et non sur des mises en récit de soi. Il ne s'agit donc pas de m'appuyer ici sur une activité narrative comme transfiguration du passé où les histoires individuelles s'organisent, s'agencent et s'imbriquent pour faire sens, mais sur des positionnements réflexifs.

\section{Le sujet et l'altérité en interaction}

Le sujet est toujours dans un va-et-vient d'altérité en interaction, de soi à l'autre mais aussi de soi à soi. De ce fait, Je n'existe que parce que l'autre le reconnaît. Ainsi, le sujet est idem, ce qu'il possède en commun avec autrui, et ipse, ce qui fait son unicité (Ricœur 1990). Dans une perspective postmoderne, le sujet naît, dans son hétérogénéité, de sa confrontation à l'altérité ; la (re)connaissance de soi passe alors par une indispensable reconnaissance de l'autre différent,

\footnotetext{
${ }^{7}$ Texte du projet.

${ }^{8}$ Ce projet, contrairement aux autres, repose uniquement sur des entretiens. Il a été financé par le Fasild et mené avec N. Auger.

${ }^{9}$ Ce projet sur la violence verbale, mené avec Véronique Fillol, était financé par la Délégation Ministérielle et a donné lieu, par la suite, à différents programmes de recherche jusqu'à aujourd'hui encore avec Béatrice Fracchiolla et Christina Romain (voir www.violenceverbale.fr)
} 
processus, à mon sens, fondamentalement et ontologiquement nécessaire pour saisir une certaine complexité et se donner du sens dans cette vie en miettes de l'expérience postmoderne (Bauman 2003, p. 310-338). La réponse au «Qui suis-je ?»s'adresse donc à un autre et se (re)trouve (un peu) par cet autre à un moment et dans une intention situés. Le sujet individuel et social n'est jamais complètement donné, il ne peut exister qu'en relation dans une quête de lui-même. Il est sujet partenaire de l'interaction avec un autre, dans le cas de ces entretiens, avec moi-même comme intervieweuse ; il ne peut se défaire du rôle social (Goffman [1974] 1993), du point de vue (Delamotte-Legrand 1999, p. 263-264), de l'ethos, ou de la « stance» (Jaffe 2009) à adopter dans les conversations ou dans les rencontres pour être et se donner à voir, dans une posture sociale et donc dans ce qu'il veut montrer de lui-même.

Or, si le « rôle » de Goffman ([1974] 1993) est pris dans la dimension sociale d'expression du sujet, la « face » en appelle à sa dimension psychique, à l'image de lui-même en accord avec l'autre mais aussi avec soi. La sociolinguistique interactionnelle et ethnographique dans la lignée de Hymes (Masquelier et Trimaille 2012) et de la Critical Discourse Analysis (entre autres Fairclough 1992, Van Dijk 2008) est exclusivement inscrite et focalisée dans l'analyse des processus sociaux. Le sujet serait alors avant tout aux prises avec les idéologies circulantes, les rapports de pouvoir et les inégalités sociales (entre autres Blommaert 1999, Dolon et Todoli 2008). Le sujet, dans ses discours, ses activités et son individuation, serait contraint pas les catégorisations, les représentations et les stéréotypes à l'œuvre, notamment à travers les variétés langagières distribuées dans des marchés légitimes ou francs (Bourdieu 1983). Dans cette optique, le sujet est alors pensé comme producteur d'identité sociale (Brès 1994) et produit de stéréotypes (Boyer 2007). Si l'on ne saurait renier de telles approches, constitutives d'une sociolinguistique interactionnelle, marquée par la pragmatique et / ou l'ethnographie de la communication dans laquelle je m'inscris pleinement, elles ne doivent pas non plus laisser dans l'ombre un sujet construit aussi par et dans l'interaction avec un autre et à travers son énonciation, et qui diraient à la fois l'être social et l'être psychique. D'ailleurs les liens entre le social et le psychisme ont largement traversé les théories du langage (Chiss et Puech 1989) et sont en filigrane par exemple à travers l'interactionnisme symbolique de Ervin Goffman, la praxématique de Robert Lafont où l'intersubjectivité, dans les traces d'Emile Benveniste. Par l'interaction se jouent à la fois la prise de position dans un rapport à l'autre et l'expression psychique du sujet, lien que je souhaite rendre visible ici.

\section{Du sujet social au sujet psychique de l'énonciation}

Le sujet se construit dans l'interaction dans un rapport à l'autre pour se dire « en regard de » 
ou « face à » dans une dimension sociale exprimée, revendiquée et / ou mise en scène. La dimension pragmatique de l'interaction ${ }^{10}$ laisse la part belle à l'intentionnalité, les buts, les actions et les bénéfices du sujet. En ce sens, les rapports de pouvoir et les stratégies discursives laissent entrevoir un sujet acteur de ses dires ou en tout cas, de sa place et de son identité sociale montrée (le sujet acteur de changement social). Certes. Mais l'énonciation est aussi « langage intérieur» (Chiss et Puech 1989, p. 9) lié à une activité dialogale, inscrite en dialogue et dialogique ${ }^{11}$, mue, entre autres, par les discours sociaux en circulation. Ainsi, l'autre interlocuteur, présent et visé, les autres, représentés et parlés, ou cet autre, double intérieur de soi, donnent à se dire au sujet, socialement et intimement à la fois tandis qu'il s'échappe à lui-même à travers boucles réflexives, lapsus, marques énonciatives, reformulations, variations stylistiques etc, bref par des franchissements intérieurs.

Parce que « le dire ne va pas de soi » (Authier Revuz 1998, p. 64), le sujet se dit aussi dans une distance à soi et utilise cette faculté réflexive que nous offre la parole. Cette «modalité autonymique », c'est-à-dire «l'activité langagière d'auto-représentation de son dire par l'énonciateur » (Authier Revuz 1998, p. 63), dit l'hétérogénéité du sujet, ses quêtes du sens, ses «prises avec la résistance des mots » (ibid. : 66), au plus juste de lui-même et dans la relation avec l'autre. Ces processus méta-énonciatifs caractérisent «l'à-dire », espace de l'inconscient, ou, du moins, de l'expression du sujet, du dire en train de se construire, au-delà d'une intentionnalité. J'aime parler de «tension $»^{12}$, qui serait, dans cette optique, ce moment de passage, cette limite, où le sujet, tendu, cherche un point d'équilibre pour être au plus juste de sa parole et de lui-même, dans son rôle social et dans sa construction psychique, face à un autre, à un $t u$. La tension énonciative rend compte d'une recherche d'équilibre où les identités se disent à travers négociations et ajustements à la fois interactionnels mais aussi dans le dire du sujet. La tension est un élément central des échanges conversationnels, qui, malgré les nécessaires coopérations, régulations et alliances, sont traversés d'engagements personnels, de défense d'opinions et donc parfois de divergences entre soi et l'autre. La tension est donc aussi à l'œuvre de soi à soi, ce qui m'intéresse ici. La tension serait cet état noué, ce besoin d'aller vers un ailleurs, un (re)nouveau du dire, pour trouver un certain équilibre discursif et

\footnotetext{
${ }^{10}$ Celle qui appréhende, entre autres, l'analyse des interactions par le biais de la speech act theory, comme c'est le cas de Eddy Roulet et de ses disciples.

${ }^{11}$ Le discours s'articule selon une double instance, un dialogue externe, dimension dialogale, entre des locuteurs différents, et un dialogue interne, dimension dialogique, qui fait écho à d'autres discours.

${ }^{12}$ La tension n'est pas tout à fait du même ordre que le paradoxe ou la contradiction. La contradiction relève de la logique discursive où des éléments argumentatifs s'excluent. Le paradoxe ne serait contradictoire qu'en apparence et rendrait des représentations du locuteur en discours en butte à ses pratiques. Il permettrait de rendre compte de situations complexes où le locuteur dans ses positionnements langagiers oscille par exemple entre représentations monolingues et unificatrices et ouverture au plurilinguisme (Forlot 2012:98)
} 
identitaire.

\section{La reformulation, la glose et les particules discursives}

Parmi les quatre espaces de «non-coïncidences du dire ${ }^{13}$ » cités par Jacqueline Authier-Revuz (1995, p. 73), les locutions, disons, c'est-à-dire, je veux dire, que j'ai choisi d'analyser ici, révèlent la non-coïncidence interlocutive, façon d'expliciter à l'interlocuteur le discours en train de se construire mais toujours dans un retour sur son propre dire et donc dans un retour à soi. C'est dans ces failles énonciatives que se révèlent les glissements du sujet pris entre une extériorité des discours et dans le temps de l'à-dire, temps de soi à soi : «En signalant parmi ces mots la présence de mots marqués comme appartenant à un autre discours, un discours dessine en lui le tracé - relevant d'une interdiscursivité représentée - d'une frontière intérieur / extérieur. Un certain nombre d'oppositions peuvent être dégagées dans l'ensemble de ces formes permettant de spécifier les types de frontières entre soi et l'autre, par lesquelles un discours produit en lui-même, par différence, une image de soi » (Authier-Revuz 1995, p. 76). Le sujet parlant est donc traversé par des voix antérieures, dans un dialogisme interdiscursif, et par des voix anticipatoires, dans un dialogisme interlocutif, des autres et de l'autre en interaction (Brès et Nowakowska 2005). Mais cette hétérogénéité des discours peut s'orienter de soi à soi, dans une forme d'interaction réflexive, dans des reprises de ce que l'on a dit et de ce que l'on va dire. Points de vue (Rabatel 2007), hypothèses, modalisation, reformulation et glose sont la manifestation autodialogique du discours qui s'inscrit dans des «boucles métaénonciatives ${ }^{14} \gg$ (Authier-Revuz 1995). Je m'intéresse donc ici, à travers les trois particules disons, c'est-à-dire et je veux dire, aux effets de reformulation.

La reformulation pourrait être définie comme «tout processus de reprise d'un énoncé antérieur qui maintient, dans l'énoncé reformulé, une partie invariante à laquelle s'articule le reste de l'énoncé, la partie variante par rapport à l'énoncé source » (Martinot 1994). On considère que l'invariance sémantique est un critère décisif pour toute reformulation. Son usage est permanent en discours tant le sujet cherche sans cesse à dire ce qu'il n'a pas assez bien dit, à

\footnotetext{
13 1. La non-coïncidence interlocutive, quand le «tu» est convoqué, «si vous voyez ce que je veux dire. 2. La noncoïncidence du discours lui même, « comme on dit au rugby ». 3. La non coïncidence entre les mots et les choses, «si on peut appeler ça une auberge ». 4. La non coïncidence des mots à eux-mêmes, «ce toit est à refaire, c'est une tuile, c'est le cas de le dire ».

14 « Les « boucles » méta-énonciatives, insérant, dans le déroulement d'une chaîne adressée par un énonciateur à un récepteur, le commentaire de l'énonciateur sur ce qu'il dit, c'est-à-dire en tant qu'il reçoit ce qu'il dit et qu'il y « répond», peuvent être considérées comme des émergence sur la chaîne de cette auto-réception sous-jacente, permanente, comme des formes par lesquelles l'énonciateur représente, ponctuellement - les «laissant apparaître » ou les mettant en scène -, la structure et le mécanisme, continuellement présents, d'un auto-dialogisme, inséparable de la distanciation interne à toute énonciation » (AuthierRevuz, 1995 : 149).
} 
préciser son argumentation, à se saisir lui-même. «Les piétinements, hésitations et bafouillages divers, parenthèses et rallonges, corrections après coup et autres repentirs, etc. [...] intéressent la problématique de la reformulation, sous l'appellation reformulation réparatrice » (Apothéloz 2008, p. 155). Il faut juger alors la reformulation ${ }^{15}$ à l'aune d'une équivalence sémantique mais aussi d'une orientation argumentative et d'une coopération conversationnelle (Martinot et Roméro 2009) qui donne place au sujet. En ce sens, il est intéressant de distinguer « les reformulations paraphrastiques qui instaurent une équivalence avec la première formulation des reformulations non-paraphrastique, lesquelles opèrent un changement de perspective énonciative » (Rossari 1997, p. 13-16).

La glose (Steuckardt et Niklas-Salminen 2003) s'inscrit aussi dans le champ de la paraphrase et de la reformulation. Au-delà d'une conception qui viserait une équivalence lexicale sémantique établie sans mobiliser un sens nouveau, sorte de définition de sens d'un mot ou d'un groupe de mots, (Steuckardt 2006, p. 6), la glose ${ }^{16}$ peut être aussi saisie dans une approche énonciative (Langhans 2003). Britta Langhans s'attache à voir en discours « ce que veut dire ce segment pour le locuteur, dans son discours concret. Les explications de mots ne se basent plus sur des équivalences lexicographiques mais sur des rapprochements situationnels, où le sens d'un terme est spécifié par des équivalences discursives » (Langhans 2003, p. 249) propres au sujet, ce qui me semble très pertinent. Ainsi, qu'on la nomme glose (groupes de mots) ou reformulation (jusqu'à des énoncés plus longs), je voudrais surtout voir, pour ma part, comment et en quoi ce phénomène, que j'appellerai reformulation, sert l'expression sociale et subjective du sujet en regard d'un autre dans un changement de perspective énonciative.

La reformulation peut s'exprimer, même si ce n'est pas toujours le cas, par des marqueurs discursifs comme ceux que j'ai choisis, disons, c'est-à-dire et je veux dire ${ }^{17}$. Les particules discursives s'inscrivent sans un processus de grammaticalisation ${ }^{18}$ mais je vais essayer de

\footnotetext{
${ }^{15}$ La reformulation a été étudiée d'abord dans un cadre syntaxique (Apothéoz 2008), conversationnel (De Gaulmyn 1987), pragmatique (pour une synthèse voir Kara 2007 et Le Bot Marie-Claude, Schuwer Martine et Richard Elisabeth 2008) puis didactique (voir entre autres Rabatel 2010). D'abord, appréhendée à partir « des marqueurs de reformulation au sens de paraphrase contextuelle ou communicative », elle a ensuite été saisie avec des marqueurs plus lâches comme ah, alors, hein où le contexte joue dans son interprétation possible (Voir Martinot et Roméro 2009).

${ }^{16}$ La glose se caractérise par les traits suivants «1) intraphrastique 2) syntaxiquement en position de parenthèse 3) parfois introduite par des indices de glose du type c'est-à-dire 4) métalinguistique 5) la capacité de se rapporter à un mot ou à un syntagme (ou groupe de mots) 6) la dimension pragmatique » (Langhans 2003, p. 247).

${ }^{17}$ On peut citer aussi pour les marqueurs de glose en dire, ça veut dire, ce qu'on appelle, comme ils disent cités par Langhans (2003) ou de façon plus générale, en d'autres termes, autrement dit, en somme, en un mot, tout bien considéré, tout compte fait, en fin de compte (Rossari 1990).

18 «Processus par lequel une forme cesse d'obéir aux restrictions grammaticales pour être contrainte par des restrictions d'ordre pragmatique et interactif » (Martel et Vincent 2001, p.142). Pour Martel et Vincent, la grammaticalisation serait à considérer selon un continuum. Les formes les plus grammaticalisées, par leur perte de sens, auraient davantage une valeur de ponctuants qui permettraient de rythmer les interactions, pour une meilleure attention dans l'échange, et de jouer, comme pour Je veux dire, " un rôle dans la structuration des énoncés et dans la gestion de l'interaction entre les interlocuteurs » (Martel et
} 
montrer, à partir de mes données, que ces marqueurs rythmiques, s'ils le sont sans doute, disent aussi une façon d'être dans son discours et tout simplement une façon d'être de soi à l'autre et de soi à soi. Je considère ces expressions d'un point de vue interlocutif et dans ce qu'elles disent d'une quête d'un dire. Il ne s'agit donc pas ici de parfaire l'étude des marqueurs ou particules discursives qui font l'objet de nombreux travaux que ce soit d'un point de vue interactionnel, pragmatique, syntaxique ou sémantique, ce qui en souligne leurs fonctions multiples en discours ${ }^{19}$. Il s'agit, par un retour sur l'élaboration d'un dire, de montrer en quoi certaines particules discursives expriment par les reformulations, une distanciation par rapport à son dire, c'est-à-dire par rapport au monde, à l'interlocuteur mais aussi par rapport à soimême dans des quêtes, des troubles, des failles du sujet qui disent ses doutes intérieurs psychiques mais aussi sociaux.

\section{C'est-à-dire, je veux dire, disons, des marques frontalières du dire ${ }^{20}$}

Dans ses études sur la reformulation, Caroline Rossari (1994), tente une description des marqueurs reformulatifs. À la différence des marqueurs argumentatifs, tels donc ou en fait les marqueurs reformulatifs auraient un effet rétroactif dans le sens où ils permettent au locuteur de revenir sur son interprétation première d'un segment discursif. Ils opèrent un « changement de perspective énonciative émanant d'un rétrointerprétation du mouvement discursif antécédent: le locuteur, suite à un première formulation donnée comme autonome et donc formant un premier mouvement discursif, en ajoute une seconde qui vient englober la première en la surbordonnant rétroactivement. Cette nouvelle formulation, présentée comme un acte principal est introduite par un connecteur reformulatif. L'usage de ce dernier permet au locuteur d'indiquer explicitement le changement de perspective énonciative opéré » (Rossari 1990, p. 346). En s'appuyant sur cette thèse, elle distingue les marqueurs qui seraient paraphrastiques, comme c'est-à-dire, sans changement de prise en charge énonciative et ceux qui seraient non paraphrastiques ${ }^{21}$. Pour elle, c'est-à-dire offrirait «par son sémantisme une relation d'équivalence entre les deux formulations [...] et si l'opération de reformulation

\footnotetext{
Vincent 2001, p. 147). Cette interprétation, pour ma part, ne me convainc pas tout à fait comme vont le montrer mes analyses qui suivent.

${ }^{19}$ Voir parmi d'autres Dostie 2004, Fischer 2006, Drescher et Frank-Job 2006, Schiffrin 1987, Vincent 1993

${ }^{20}$ Pour l'étude de ces marqueurs voir aussi d'un point de vue variationniste (Beeching 2007), pragmatique (Khachaturyan 2008, Martel et Vincent 2001, Tremblay 1998) et énonciatif (Langhans 2003, Rossari 1990, Teston-Bonnard 2008, Vassiliadou 2004).

${ }^{21}$ Parmi les marqueurs rerformulatifs non périphrastiques, elle mentionne ceux qui permettent 1. la récapitulation (en somme, bref, en un mot) avec une faible prise de distance, juste expression plus condensée. 2. l'invalidation (enfin) avec une prise de distance très forte. Invalider rétroactivement un aspect de sa première formulation (« tu devrais aller voir Pierre, enfin tu fais ce que tu veux »). 3 la distanciation (de toute façon, de toute manière, en fait, de fait, en réalité, en tout cas, au fond) avec une forte prise de distance mais sans invalidation. 4 la reconsidération (tout bien considéré, tout compte fait, somme tout, après tout).
} 
paraphrastique permet au locuteur de revenir sur un énoncé antécédent par l'intermédiaire d'un connecteur reformulatif, ce n'est pas dans le but d'exprimer dans la reformulation un changement de perspective énonciative. Au contraire, le locuteur utilise cette opération pour revenir sur sa première formulation afin de la compléter, la clarifier ou même la rectifier, tout en instaurant avec celle-ci une équivalence à quelque niveau que ce soit » (Ibidem p. 348349). » À partir des extraits des entretiens que j'ai analysés, je voudrais montrer que les stratégies $^{22}$ énonciatives, exprimées dans les marqueurs de reformulation en dire, notamment c'est-à-dire, et à l'encontre de l'hypothèse de Rossari citée ci-dessus et reprise par la suite (notamment Vassiliadou 2004), sont multiples et rendent compte de procédés argumentatifs et de positionnements énonciatifs pluriels et réflexifs.

Ces différentes fonctions de la reformulation, au-delà de celles attendues et les plus facilement catégorisables, l'élaboration discursive (la recherche du mot juste, le recentrage thématique, la caractérisation définitoire), les stratégies argumentatives, décèlent un positionnement énonciatif du sujet, positionnement fait de tensions discursives, pour se dire comme sujet social et psychique, en allant puiser à l'intérieur de soi, entre flottements et frottements.

\section{L'élaboration discursive}

Une première analyse permet de voir que les particules de reformulation servent le fil discursif, avec explication, clarification ou rectification, au plus juste de ce que veut faire entendre le locuteur, mais une deuxième analyse montre que le sujet se déplace aussi dans son dire.

\section{La recherche du mot juste}

Dans l'exemple 1, Noël exprime son désappointement face à l'action politique en contexte minoritaire.

Exemple 1.

\begin{tabular}{|l|l|}
\hline 1 & N : puis on a essayé d'embarquer là dedans puis ça ben on s'est fait tasser avec: à force \\
\hline 2 & de: enfin + par un avocat quoi t'sais y était rendu que: t'sais: y nous: on était rendus là \\
\hline 3 & t'sais que c'étaient les avocats qui nous montraient la porte ça beh j'ai dit bon beh si \\
\hline 4 & c'est ça + on a sacré notre camp de là mais ça ça m'a un peu: + disons quand tu parles \\
\hline 5 & de de vouloir faire de l'action politique là ça m'a ça m'a fait déchanter t'sais quand je \\
\hline 6 & me suis rendu compte à quel point là c'est: c'est plat t'ça X devoir là: jouer des parties \\
\hline 7 & de bras de fer avec des gens là qui qui aiment pas qui tu es juste pour commencer puis \\
\hline 8 & que à cause de ça t'sais i comprend pas tes idées + \\
\hline
\end{tabular}

\footnotetext{
22 « La stratégie ne saurait correspondre aux buts conscients que se fixe le sujet et ne saurait caractériser son action de manière autonome. Afin d'éviter le problème épineux de la conscience (intentionnalité ?), il serait préférable d'analyser la stratégie comme un comportement communicatif constaté et analysé une fois l'interaction terminée, enregistrée et transcrite. La stratégie est donc une construction de l'analyste qui, s'appuyant sur les activités langagières et mise en scène des divers types de places construites par les sujets, s'efforce de rendre compte des lignes d'actions suivies » (Vion 1998, p. 200)
} 
Dans l'exemple 1 disons $^{23}$ (l. 4) sert à donner le temps d'élaborer le sens en discours, de trouver le mot juste «action politique ». Dans cet exemple, en particulier, il est remarquable de voir combien d'autres particules (et leur répétition) contribuent à l'effet produit par disons et à sa fonction : t'sais, beh, bon beh. Noël montre combien la mobilisation politique en milieu minoritaire se trouve confrontée à des oppositions majoritaires. La particule discursive disons permet, face à la quête impossible du mot juste ("ça m’a un peu: + », l. 4), de (re)formuler dans une "élucidation du contenu » (Langhans 2003), comme le marque la pause précédant disons (l. 4). Le changement de construction syntaxique (passage d'un adjectif possible (l. 4) à une proposition «ça m'a fait déchanter », l. 5), permet à Noël d'exprimer plus facilement la déception vécue face à l'action politique, alors que les avocats ne soutiennent pas jusqu'au bout les démarches entreprises par les militants.

Ce qui est intéressant, c'est de voir que cette élaboration du thème est au cœur de la constitution de cet acteur en tant que sujet, puisqu'elle lui permet de basculer sur l'expression « ça m’a fait déchanter (l.5) », désenchantement au cœur de son discours, qui s'actualise encore en « je me suis rendu compte à quel point là (l.6)». L'explicitation discursive par la reformulation permet au sujet d'exprimer non seulement des changements de position en tant qu'acteur social mais des doutes personnels en «je ». Même si l'on peut considérer cette élaboration comme un piétinement discursif, on voit que disons sert à rendre plus adéquat le dire de Noël, à l'ajuster pour éviter toute ambiguïté à l'égard de son interlocutrice et de se positionner aussi comme militant en situation minoritaire donc dominée (« avec des gens là qui aiment pas qui tu es juste pour commencer puis que à cause de ça t'sais i comprend pas tes idées ») et d'affirmer ses propres tensions. C'est de cette façon que je voudrais signifier les frontières sociolinguistes, celles qui rendent compte de oscillations intérieures, de passages de soi au sujet social.

Dans l'exemple numéro 2, Noël cherche à identifier le mode de communication qu'il sent comme peu pertinent pour les projets franco-ontariens.

Exemple 2.

\begin{tabular}{|l|l|}
\hline 1 & N : je moi j'ai j'ai le sentiment que: des projets pour faire avancer les projets là c'est tu \\
\hline 2 & peux pas passer par euh euh + disons des des communications faites par les médias t'sais \\
\hline 3 & faut + les gens réagissent pas à ça les gens s'attendent à être joints là sur une base \\
\hline 4 & individuelle entre quatre: yeux là + puis le contact euh + à chaud là tu vois ben c'est pas \\
\hline
\end{tabular}

${ }^{23}$ À partir d'une analyse de Catherine Chanet (2004) autour du Corpus de Référence du Français Parlé (450 000 mots) et du CORPAIX (1 050000 mots), Disons serait la $25^{\mathrm{e}}$ particule discursive la plus employée sur les 85 choisies et apparaîtrait en moyenne toutes les $17 \mathrm{mn} 26 \mathrm{~s}$. 


\begin{tabular}{|l|l|}
\hline 5 & possible + on on on (n') est pas comme une masse + les Franco-ontariens on est des on est \\
\hline 6 & des petites poches de gens là qui se connaissent entre eux puis qui s'attendent à se \\
\hline 7 & connaîsse à se connaître entre eux là t'sais \\
\hline
\end{tabular}

Le disons sert de glose explicative, dans un effet d'approximation, d'hypothèse soumise à l'interlocuteur. Il permet de préciser le contenu sémantique d'un mot ou d'un groupe de mots énoncés. Mais, plus que ça, par là-même Noël peut s'inscrire dans son discours et prendre une position subjective, donner son propre avis qui va à l'encontre de la communication attendue, celle « faite par les médias » (l.2). Cette reformulation montre, comme dans l'exemple 1, la prise de position comme acteur social en tant que représentant de sa communauté (il sait ce qu'il convient de faire, " on on on (n') pas comme une masse + les Franco-ontariens on est des on est des petites poches de gens » (l.5 et l.6) mais aussi comme sujet («j'ai le sentiment» (l.1) pris entre deux plans possibles d'action, dont l'un évoqué à travers la reformulation ne pourra qu'être rejeté.

Ainsi, au-delà d'une volonté de précision et d'explicitation pour l'interlocuteur à travers la recherche du mot juste, ces reformulations permettent au sujet d'exprimer une position énonciative subjective par un déplacement du point de vue, entre expressions d'une déception (exemple 1) ou d'une minorisation (exemple 2), expressions qui vont bien au-delà d'une simple reformulation sémantique. Ces ancrages énonciatifs marquent un balancement entre un positionnement social et une tension individuelle.

\section{Le recentrage thématique}

Dans l'exemple 3, Noël parle d'un changement de politique éditoriale de la maison d'édition dans laquelle il travaillait.

Exemple 3

\begin{tabular}{|l|l|}
\hline 1 & N : Prise de parole disons du temps que j'y étais là j'ai l'impression que le gros virage: a \\
\hline 2 & été pris à un moment donné là quand la la directrice actuelle est arrivée ce::: qui a fini par \\
\hline 3 & se mettre en place c'est de vraiment euh: + miser sur la distribution de ces ouvrages là \\
\hline 4 & selon les canaux le: commerciaux classiques \\
\hline
\end{tabular}

La précision discursive peut se manifester aussi à travers une incise (« disons du temps que j'y étais », l.1), parenthèse restrictive, dans une forme d'hypothèse et de précaution argumentative. Noël précise que le développement de la maison d'édition s'est bien produit mais, d'après ce qui lui semble, dans le temps où il était lui-même en fonction dans la structure. Il exprime par l'incise en « je » sa place d'acteur social puisque, pendant le temps 
où il travaillait à la maison d'édition Prise de parole, il a vu des changements en faveur du développement de la diffusion d'auteurs franco-ontariens, donc en faveur d'une certaine visibilité de la minorité. Là encore, cette précision ne sert pas seulement l'interlocuteur mais aussi le positionnement du sujet, de Noël lui-même, qui dans une boucle réflexive, montre combien le changement social vécu est lié à sa perception et à son expérience.

\section{La caractérisation définitoire}

Dans l'exemple 4, pour Noël, être francophone serait avoir un certain avantage professionnel. Exemple 4.

\begin{tabular}{|l|l|}
\hline 1 & $\mathrm{~N}$ : c'est pas sûr que j'aurais eu un si beau euh trajet professionnel: ailleurs qu'à Sudbury \\
\hline 2 & puis: en tant que francophone disons ça donne cet avantage on est disons moins \\
\hline 3 & nombreux à se à se à se battre pour les les mêmes postes là donc \\
\hline
\end{tabular}

Ce disons offre une glose sémantique, une forme de caractérisation définitoire, mais qui, audelà de la reformulation, positionne le sujet comme acteur social (« on est disons moins nombreux à se à se battre pour les les mêmes postes donc » $(l .2$ et $l .3)$.

De la même façon, Hassan explique, dans l'exemple 5, la fermeture des «frontières » en la liant au front national.

Exemple 5.

\begin{tabular}{|l|l|}
\hline 1 & H/ maintenant y a y a à peu près quinze ans + euh on a fermé les frontières on on disons \\
\hline 2 & on: y avait une vague: avec le front national et tout ça sur les immigrés et maintenant on \\
\hline 3 & se rend compte que ((bruits de poule $)$ on a fermé les frontières et peut-être il vaut mieux \\
\hline 4 & faire appel encore à des immigrés ((bruits de tasse $))$ \\
\hline
\end{tabular}

Dans cet exemple, la reformulation qui s'étend sur toute l'argumentation ( $l$. 2 à $l$. 4) permet à Hassan à la fois de donner son point de vue social sur la question des frontières et de se positionner en tant qu'immigré lui-même.

Dans les exemples 4 et 5 , les reformulations permettent de revenir sur des notions centrales dans la définition de soi et du groupe, celles de « francophone » et de « frontières ». Dans une rétro-interprétation, les locuteurs changent la perspective énonciative attendue et dans un « on » inclusif dialogique (« on est moins nombreux », « on se rend compte ») donnent force à une prise de position en contre point, « quand être francophone a aussi des avantages », ou « quand fermer des frontières a aussi des inconvénients ».

Dans les exemples 6 et 7, Hassan répond à la question de savoir si la double culture est un 
élément positif et s'il a des frères et sœurs.

Exemple 6.

\begin{tabular}{|l|l|}
\hline 1 & H : et euh: j'en suis certain ça posera aucun problème pour les enfants parce que / euh \\
\hline 2 & disons je travaille beaucoup donc j'ai un: ça va j'ai un: bien donc je je il manque rien du \\
\hline 3 & tout: j'ai plein de trucs euh euh ma femme aussi et un jour je pense que je vais vivre au \\
\hline 4 & Maroc \\
\hline
\end{tabular}

\section{Exemple 7}

$1 \quad$ H : ouais j'ai un j'ai un frère aussi qui a fait maths sup maths spé et c'est disons c'est pas 2 l'immigration euh main d'œuvre

Hassan revient, dans les exemples 6 et 7, à ce qui le touche dans son identité sociale («je travaille beaucoup » (l.2, ex.6), «c'est pas l'immigration euh main d'œuvre » (l.2, ex 7) mais les arguments donnés pour convaincre de sa position sociale sont ponctués par de nombreux piétinements, une voix en « je » dont les disons signifient aussi les fluctuations de ses doutes intérieurs. Les modalisateurs et les hésitations montrent que les déductions définitoires (ce qui n'est pas problème pour les enfants, ce que c'est que maths spé) données seraient une possibilité d'explication parmi d'autres face aux balancements intérieurs.

Dans l'exemple 8, Fatima montre, par la définition de ce que signifie « l'ordre du psy », ce qui doit être donné à un enfant de couple mixte pour être serein.

Exemple 8

\begin{tabular}{|l|l|}
\hline 1 & F : non mais moi je je enfin si tu veux que ça se joue beaucoup au niveau de: de quelque \\
\hline 2 & chose qui est de l'ordre du psy c'est-à-dire que là-dessus on peut développer pendant des \\
\hline 3 & heures hein + à savoir que: + ((bruit)) un je pense qu'un gamin pour qu'il soit \\
\hline 4 & relativement bien dans ses pompes il faut qu'il sache un peu d'où il vient + ça ça me \\
\hline 5 & paraît primordial en termes de transmission etc \\
\hline
\end{tabular}

Fatima explique le « quelque chose qui est de l'ordre du psy » (l.2) avec le c'est-à-dire qui permet une élaboration du sens qui ne va pas de soi («enfin si tu veux» (l.1) «on peut développer pendant des heures hein » (l.2), «je pense» (l.3). Toutefois cette définition entraîne, dans des glissements, un positionnement énonciatif centré en « je » en lien, toutefois avec un engagement social, une volonté de transmission culturelle par le nom. Les retours sur soi, au-delà des positionnements en tant qu'acteur social, s'accompagnent bien souvent de marques d'hésitations, de reprises, de piétinements assez nombreux (exemples 6, 8 et suivants $9,10,12)$ qui signifient les expressions de soi faites de quelques vacillations.

\section{Les stratégies argumentatives}


Une autre façon d'aborder l'analyse est de saisir ces particules reformulatrices par l'argumentation. Elles servent alors à entraîner l'adhésion de l'interlocuteur quand le développement explicatif se rassemble dans un effet conclusif. «Les commentaires métadiscursifs évaluatifs ont un effet immédiat sur la conscience de l'interlocuteur, le guidant dans une interprétation. De fait, plutôt que de fournir des munitions à l'interlocuteur, on les lui enlève » (Martel et Vincent 2001 p. 144). Mais, encore une fois, l'argumentation permet davantage, la faculté pour le sujet de se dire comme acteur social voire dans sa complexité personnelle.

Dans l'exemple 9, Fatima soutient que porter deux prénoms est une pratique de plus en plus courante.

Exemple 9.

\begin{tabular}{|l|l|}
\hline 1 & F : et d'ailleurs dans la tradition arabe c'est E: le nom du père si tu veux qui E: + enfin + \\
\hline 2 & moi je pense qu'elles pourraient elles pourraient porter les deux les deux noms plus tard \\
\hline 3 & $(($ bruits $)$ plus grandes mais que:: petites effectivement c'est à la fois c'est notre je veux \\
\hline 4 & dire c'est c'est: \\
\hline 5 & P : mais c'est pas une tradition en plus ici \\
\hline 6 & F : enfin chez nous si c'est: je non \\
\hline 7 & C : les deux noms non ah non \\
\hline 8 & P : les deux noms c'est pas une tradition \\
\hline 9 & C : chez nous non absolument pas \\
\hline 11 & C : bof \\
\hline 12 & F : ça se fait ça se fait disons +++ \\
\hline
\end{tabular}

L'emploi du disons (l.12) conclusif lui permet de clore l'échange en affirmant sa position et croire par là même entraîner l'adhésion de ses interlocuteurs. Dans ce cas là, ces visées argumentatives permettent d'asseoir des prises de positions identitaires où l'acteur social s'affirme dans ses engagements. En même temps, on voit bien que Je veux dire (l. 3-4), très souvent utilisé dans mes données, permet l'élaboration discursive, une affirmation identitaire, un engagement social mais aussi un balancement personnel entre différents choix possibles, celui ici de donner un ou deux prénoms, balancement entre un «chez nous » et un « ici », avant de clore, avec le « disons » (l.12), les choix discursifs dans une interaction conclusive.

Dans l'exemple 10, Fatima montre le poids des discours du Front National auprès de ses enfants.

Exemple 10

$1 \quad$ F : oui non + oui non + oui non parce que: y a des moments euh: il suffit qui y ait 


\begin{tabular}{|l|l|}
\hline 2 & quelques tensions si tu veux au niveau social bon là c'est vrai que + j'ai l'impression \\
\hline 3 & qu'on les vit moins mais il suffit qui y ait un + je me souviens par exemple des moments \\
\hline 4 & où:: (bruits) où le Front National avait j'sais pas combien où y avait plein de débats là- \\
\hline 5 & dessus etc je veux dire bon euh: + ((raclement de gorge)) c'est au niveau de des enfants \\
\hline 6 & ça veut dire que c'est pas forcément des débats euh + très profonds et tout mais euh: bon \\
\hline 7 & les filles comprenaient bien ce que ça voulait dire je veux dire l'histoire de Le Pen et \\
\hline 8 & compagnie + on avait quand même des débats là-dessus et euh: bon elles faisaient des \\
\hline 9 & observations et tout parce que \\
\hline
\end{tabular}

Dans le même sens, ici le Je veux dire engage le sujet dans une prise de distance par rapport à son propre dire. Fatima mentionne, dans l'exemple 10, par le premier je veux dire (l. 5) le fait que les débats autour du Front National ne sont pas inoffensifs et peuvent peser sur ses enfants. Ce positionnement est à la fois social mais également personnel et familial quand le second je veux dire (l.7), renvoie avec quelques reprises et hésitations puis dans une glose autour du « ce que ça voulait dire » (l.7), à « l'histoire de Le Pen et compagnie » (l.7-8) et à sa propre identité qui pourrait être malmenée.

Dans l'exemple 11, Juan, en tant que conseiller d'éducation, donne son avis sur la situation éducative au sein d'un collège.

Exemple 11

\begin{tabular}{|l|l|}
\hline 1 & J.alors et et ça devient et ça devient vraiment: + grave: quand quand tout le monde \\
\hline 2 & dramatise + pas le gosse mais tout l'entourage + en faire un drame ça l'arrange pas / faut \\
\hline 3 & marquer le coup souvent euh faut marquer le coup mais non c'est faux j'veux dire en \\
\hline 4 & rigoler et lui dire mais mais t'sais très bien que ça se fait pas parce qu'au fond i(ls) le \\
\hline 5 & de de vouloir faire de l'action politique là ça m'a ça m'a fait déchanter t'sais quand je \\
\hline 6 & savent très bien + mais si t'en fais un drame après quan(d) i(l) va sortir de du du bureau ou \\
\hline 7 & quand après avoir pris euh: trois jours d'exclusion i(l) va dire à ses copains ah moi j'ai: \\
\hline 8 & seize jours d'exclusion et toi: dix-huit ah: t'vois tu me bats et tout / voilà c'est c'est la fierté \\
\hline 9 & c'est même plus d'avoir la meilleure note c'est d'avoir pour voir celui qui a fait le plus le \\
\hline 10 & con / \\
\hline
\end{tabular}

À partir de « en rigoler », Juan montre qu'il s'oppose à la doxa éducative qui affirme devoir « marquer le coup » $(l .3)$ quand un élève a commis une faute. Il développe son «c'est faux » par « en rigoler» (l.3 et l.4) et par toute la mise en narration qui suit (l.6-8). Le je veux dire lui permet d'argumenter dans une prise de position distanciée marquée par une élaboration de son dire (hésitations, ruptures, reprises, narration...) qui dit son questionnement par rapport aux discours circulants. Sa prise de parole se caractérise par des boucles réflexives mais exprime aussi sa position «politique », comme acteur social désireux de changement.

Dans l'exemple 12, Noël explique d'où vient son lien avec le français.

Exemple 12. 


\begin{tabular}{|l|l|}
\hline 1 & F : ça fait qu'ce côté là ç'a a pas fonctionné comme ma mère aurait voulu là mais + \\
\hline 2 & mes parents eux viennent de Jogues + ça c'est un p'tit village au sud de Hearst + qui \\
\hline 3 & est très français + \\
\hline 4 & Enq : mm \\
\hline 5 & N :comme Hearst l'est d'ailleurs + puis E: + eux ont grandi là j'pense que: en fait \\
\hline 6 & mon père est né là ma mère elle: + j'pense qu'elle est arrivée là à l'âge d'un ou \\
\hline 7 & deux ans très jeune + de Montréal c'était à l'époque de la colonisation là d'ce coin \\
\hline 8 & de pays là + ça fait que eux ont grandi là puis c'est: $E:++$ c'est sans doute pour ça \\
\hline 9 & beh que disons que le français ça fait partie de mon identité puis que j'y tiens c'est \\
\hline 10 & que donc finalement ils le sont c'est $E:+$ \\
\hline
\end{tabular}

Disons peut s'accompagner alors d'un élément argumentatif («c'est sans doute pour ça » l.8), où Noël explique en quoi son attachement au fait français est lié à l'histoire de ses parents. Il développe d'abord son argumentation en montrant que la situation géographique (habiter un village du Nord très français) et historique (le temps de la colonisation francophone souhaitée pour conquérir des terres anglaises) a joué dans son lien avec le français, donc a construit son appartenance sociale. Le disons (l.9), avec le sans doute (l.8), sert par la suite, à modaliser l'explication univoque sociale, en mettant le sujet au cour de son dire en proposant une explication en forme d'hypothèse identificatoire « le français ça fait partie de mon identité puis que j’y tiens » (l.9), annoncée avec précaution par « c'est sans doute pour ça beh que»(l.8). Ainsi, quand le sujet touche à ce qui le constitue, le développement argumentatif, extérieur à soi (la situation géographique et historique liée à ses parents) permet à Noël de revenir à lui-même, («mon identité », «j’y tiens » (l.9). On voit bien ici comment Disons sert alors à « confronter son propos aux autres possibles » (TestonBonnard 2008, p. 62) en affirmant, dans une construction discursive à la fois le rôle social puis personnel du sujet.

\section{En guise de conclusion}

Les trois particules de reformulation en dire que j'ai essayé d'analyser, disons, c'est-dire, je veux dire, à partir des données extraites d'entretiens sociolinguistique réalisées au cours de différentes recherches, s'inscrivent, sans récurrence systématique et sans distinction caractérisée, dans des nécessités explicatives et argumentatives. À travers élaborations discursives ou stratégies argumentatives, elles permettent certes de préciser le fil du discours, à un interlocuteur qu'il faut convaincre de positionnements identitaires, mais également de laisser le sujet affirmer des positionnements sociaux et donc des participations à des engagements et des changements importants (mobilisation communautaire ou migration par 
exemple). Ces particules à travers l'expression de situations personnelles, (profession, fonction parentale, enfance), invitent, en même temps, à des rétro-interprétations qui constituent le sujet au plus intime de lui-même, ce que je voudrais définir comme « frontières sociolinguistiques », balancements intérieurs, de soi au sujet social.

\section{Conventions de transcription}

- Soulignement $=$ chevauchement

- $\quad+\quad=$ micro-pause

- exTRA = segment accentué, majuscules

- $(($ rires $))=$ phénomènes non transcrits,

- : = allongement vocalique

- (il) y a / i(l) dit = segment non réalisé

- $\operatorname{xxx}=$ segment incompréhensible

\section{Références bibliographiques}

Apothéloz Denis, 2008, «Reformulations réparatrices à l'oral», La reformulation. Marqueurs linguistiques, stratégies énonciatives, M.-L. Le Bot, M. Schuwer, Richard R.Elisabeth éd., Rennes, Presses Universitaires de Rennes, p.155-168.

Authier Revuz Jacqueline, 1995, Ces mots qui ne vont pas de soi. Boucles réflexives et noncoïncidences $d u$ dire, Paris, Larousse, Authier Revuz Jacqueline, «Enonciation métaénonciative. Hétérogénéités énonciatives et problématiques du sujet », Les sujets et leurs discours. Enonciation et interaction, R. Vion éd., Aix-en-Provence, Université de Provence, 1998, p. 63-80.

Barth Frederik, "Introduction», 1969, Ethnic Groupes and Boundaries. The Social Organization of Culture Difference, F. Barth éd., Boston, Little, Brown and Co., p. 9-38.

Bauman Zygmunt, , 2003, [1995], La vie en miettes. Expérience postmoderne et moralité, Chambon, Le Rouergue, Life in Fragments, Oxford, Backwell Publishing.

Benveniste Emile,1974, Problème de linguistique générale, Paris, Gallimard.

Beeching Kate, 2007, «La co-variation des marqueurs discursifs bon, c'est-à-dire, enfin, hein, quand même, quoi et si vous voulez : une question d'identité ?», Langue française numéro 154, p. 78-93, http://www.cairn.info/revue-langue-francaise-2007-2-page-78.htm

Blommaert Jan (Ed), 1999, Language Ideological Debates, Berlin, New-York, Mouton de Gruyter.

Bourdieu Pierre, 1983, «Vous avez-dit «populaire»? L'usage de la parole, Actes de la Recherche en Sciences Sociales, n46, Paris, Minuit, p.98-105

Boyer Henri éd, 2007, Stéréotypage, stéréotypes fonctionnements ordinaires et mises en 
scène, 4 volumes, Paris, L'Harmattan.

Brès Jacques, 1994, Récit oral et production d'identité sociale, ville, Presses de l'université de Montpellier 3.

Brès Jacques et Nowakowka Aleksandra, 2005, « Dis-moi avec qui « tu dialogues » je te dirai qui tu es... De la pertinence de la notion de dialogisme pour l'analyse de discours », Analyse de discours. Etats de l'art et perspectives. Marges Linguistiques n9, p.137-154

http://www.revue-texto.net/Parutions/Marges/00 ml092005.pdf

Chanet Catherine, 2004, «Fréquence des marqueurs discursifs en français parlé : quelques problèmes de méthodologie », Recherches sur le français parlé, n¹8, p. 83-106.

Chiss Jean-Louis et Puech Christian, 1989, «Enonciation, interaction et conversation : les théories du langage entre le psychisme et le social», HEL, 11, 2, p. 7-36.

De Gaulmyn Marie-Hélène, 1987, «Reformulation et planification métadiscursive », J. Cosnier et C. Kerbrat-Orecchioni, Presses universitaires de Lyon, p. 167 -198.

Delamotte-Legrand Régine, 1999, «Les pairs sont-ils des mêmes ou des autres ? », J. Bres, R. Delamotte-Legrand, F. Madray-Lesigne et P. Siblot éd, L'autre en discours, Presses universitaires de l'université Montpellier 3, p. 261-285.

Dolon Rosana et Todoli Julia éd, 2008, Analyzing Identities in Discourse, Amsterdam, Philadelphia, John Benjamins.

Dostie Gaétanne, 2004, Pragmaticalisation et marqueurs discursifs. Analyse sémantique et traitement lexico-graphique, Bruxelles, Editions Duculot.

Drescher Martina et Frank-Job Barbara éd, 2006, Les marqueurs discursifs dans les langues romanes. Approches théoriques et méthodologiques, Frankfurt, Peter Lang.

Fairclough Norman, 1992, Discourse and Social Change, Cambridge, Polity.

Fischer Kerstin éd, 2006, Approaches to discourse Particles, Amsterdam Elsevier.

Forlot Gilles, 2012, Pratique et représentation de la diversité linguistique, entre francophonie et anglophonie, perspectives sociolinguistiques sur l'appropriation des langues, Habilitation à Diriger des Recherches, Université de Picardie.

Goffman Erving, [1974] 1993, Les rites d'interaction, Paris, Minuit.

Heller Monica, 2002, Eléments d'une sociolinguistique critique, Paris, Didier.

Hymes Dell, 1972, « Models of the interaction in social life », J. Gumperz and D. Hymes éd Directions in sociolinguistics, New York, p. 35-71

Jaffe Alexandra éd, 2009, Stance : Sociolinguistics Perspectives, Oxford University Press.

Kara Mohammed éd, 2007, Usages et analyses de la reformulation, Recherches linguistiques $\mathrm{n}^{\circ} 29$, Université de Metz. 
Khachaturyan Elisaveta, 2008, «Les mots du discours de dire », Estudos Linguisticos / Linguistic Studies, CLUNL, Lisboa, p. 287-315

Langhans Britta, 2003, «Indices de gloses dans un corpus d'entretiens », A. Steuckardt et Niklas-Salminen A. éd, le mot et sa glose, Publications de l'Université de Provence, p. 245271.

Lave Jean et Wenger Etienne, 1991, Situated Learning: Legitimate Peripheral Participation, Cambridge, Cambridge University Press.

Le Bot Marie-Claude, Schuwer Martine et Richard Elisabeth éd, 2008, La reformulation. Marqueurs linguistiques, stratégies énonciatives, Presses Universitaires de Rennes.

Martel Guylaine et Vincent Diane, 2001, «Particules métadiscursives et autres modes langagières : des cas de changement linguistique », Revue Travel, 34-35, p.141-152

Martinot Claire, 1994, La reformulation dans les productions orales de définitions et explications, Thèse de doctorat, Paris 8 .

Martinot Claire et Roméro Clara, 2009, « La reformulation : acquisition et diversité des discours », C. Martinot Claire et C. Roméro éd, La reformulation, Université Montpellier 3, Cahiers de praxématique, p. 7-18.

Masquelier Bertrand et Trimaille Cyril éd, 2012, Dell Hymes : héritages et débats, Langage et Société, $\mathrm{n}^{\circ} 139$.

Rabatel Alain, 2007, « Analyse énonciative du point de vue, narration et analyse de discours », Université de São Paulo, Filologia e Linguistica Portuguesa 9, p. 345-368.

Rabatel Alain éd, 2010, Les reformulations pluri-sémiotiques en contexte de formation, Presses Universitaires de Franche-Comté.

Ricœur Paul, 1990, Soi même comme un autre, Paris, Seuil, Points Essais.

Rossari Corinne, 1990, Cahiers de linguistique française, $\mathrm{n}^{\circ}$ 11, p. 345-359.

Rossari, Corinne, 1994, Les opérations de reformulation, Berlin Peter Lang.

Schiffrin Deborah, 1987, Discourse Markers, Cambridge University Press.

Simonin Jacky et Warthon Sylvie éd, 2003, Sociolinguistique du contact. Dictionnaire des termes et concepts, Lyon, ENS Editions.

Steuckardt Agnès, 2006, « Du discours au lexique : la glose », séminaire de l'Atilf. http://www.atilf.fr/IMG/pdf/seminaires/Seminaire_atilf_Steuckardt_2006-03.pdf

Steuckardt Agnès et Niklas-Salminen A. éd, 2003, le mot et sa glose, Publications de l'Université de Provence.

Teston-Bonnard Sandra, 2008, « Je veux dire est-il toujours une marque de reformulation ? » Le M.-C. Bot, M. Schuwer et E. Richard éd, La reformulation. Marqueurs linguistiques, 
stratégies énonciatives, Presses Universitaires de Rennes, p. 51-69.

Tremblay Judith, 1998, Etude de quelques marqueurs discursifs dans le français oral du Québec : disons et mettons, Mémoire de Maîtrise, Univerité du Québec à Chicoutimi.

Vassiliadou Hélène, 2004, «Les connecteurs de reformulation c'est-à-dire en français et diladi en grec », Lingvisticoe Investigationes 27 (1), p. 125-146.

Vincent Diane, 1993, Les ponctuants de la langue et autres mots du discours, Québec, Nuit Blanche Editeur.

Van Dijk Teun A., 2008, Discourse and Power, New York, Palgrave Macmillan.

Vion Robert, 1998, «Le sujet en linguistique », Les sujets et leurs discours. Enonciation et interaction, R. Vion. éd, Presses de l'université de Provence, p.189-202. 